\title{
Ungleichheit, Intersektionalität und Alter(n) - für eine räumliche Methodologie in der Ungleichheitsforschung
}

\author{
Friederike Enßle and Ilse Helbrecht \\ Geographisches Institut, Humboldt-Universität zu Berlin, Berlin, Germany \\ Correspondence: Friederike Enßle (friederike.enssle@hu-berlin.de)
}

Received: 18 December 2017 - Revised: 13 June 2018 - Accepted: 20 August 2018 - Published: 13 September 2018

Kurzfassung. This article explores the interplay of inequality, space and age(ing) from an intersectional perspective. It argues, that a spatial research methodology is most fruitful in order to include age(ing) in debates on intersectionality and inequality. Drawing on geographical gerontology and inequality research, we scrutinize the research gap at the intersection of these fields: While age is a neglected factor in intersectional debates on inequality, questions of power are hardly addressed by geographical gerontology. To bridge this research gap, we propose space as methodological perspective. By showing how the negotiation of age(ing) varies in different spatial settings, the article emphasises the value of spatial approaches to analyse the two faces of age(ing) - age as a marker of difference and ageing as a process. On a larger scale, the article points to the potential of a spatial methodology to approach the complexity of intersectionality.

\section{Einleitung}

Soziale Ungleichheit und gesellschaftliche Alterung zählen zu den drängenden Herausforderungen der Gegenwart. Jedoch befassen sich aktuelle Debatten in Politik und Gesellschaft entweder nur mit wachsenden Einkommensunterschieden und der ungleichen Verteilung von Ressourcen oder mit den Herausforderungen des demographischen Wandels und der alternden Gesellschaft (Helbrecht, 2013). Zwar haben in den letzten Jahren auch die ungleichen Lebenslagen älterer Menschen an Aufmerksamkeit gewonnen (vgl. Künemund und Schroeter, 2008; Mahne et al., 2017) und insbesondere Einkommensunterschiede werden unter dem Stichwort Altersarmut vielfach diskutiert (vgl. Vogel und MotelKlingenbiel, 2013). Allerdings werden die Überschneidungspunkte der beiden Phänomene nur selten betrachtet. Und so bleibt das Alter in seiner Diversität im Vergleich zu Geschlecht, Ethnizität, sozialer Klasse oder Sexualität ein wenig beachtetes Feld der sozialwissenschaftlichen Forschung zu Ungleichheit. Das ist insofern erstaunlich, als mit der wachsenden Zahl älterer Menschen auch mehr Angehörige von Minderheiten und vulnerablen Gruppen ein höheres Alter erreichen. Forschung zu sozialer Ungleichheit oh- ne die Berücksichtigung des Alters übersieht einen großen und wachsenden Teil der Gesellschaft. Was die Differenzkategorie Alter in Relation zu anderen Merkmalen bedeutet und welchen konzeptionellen Mehrwert das Alter Debatten um Ungleichheit hinzufügen kann, ist bislang nur wenig diskutiert worden.

In unserem Beitrag greifen wir diese zentrale Auslassung der Forschungsliteratur auf und plädieren für eine humangeographische Auseinandersetzung mit Alter als einer relationalen Kategorie der Ungleichheit. Dabei sehen wir den Raum als Prisma, durch welches das Zusammenspiel von Ungleichheit und Alter in seiner Komplexität sichtbar und für empirische Untersuchungen und konzeptionelle Auseinandersetzungen zugänglich gemacht wird. Als relationale Kategorie steht das Alter - wie alle Differenzkategorien nicht für sich allein, sondern wird in Beziehung zu anderen strukturellen Merkmalen wie Geschlecht, Ethnizität, sozialer Klasse, Sexualität oder Religion unterschiedlich wirksam. Das Alter kann also nur in Relation zu anderen Faktoren, sprich als relationale Kategorie, verstanden werden. Darüber hinaus ist das Alter auch in seiner Eigenschaft als lebenslanger Prozess relational zu begreifen. Der Altersprozess wird erst in Beziehung zu Menschen anderer Altersstu- 
fen und zum eigenen Lebensverlauf wahrnehmbar (van Dyk, 2015a). Die Gleichzeitigkeit von Alter als Strukturkategorie und Prozess unterscheidet das Alter von anderen Differenzkategorien. Der stetige Wandel der Kategorie Alter - das Altern - verdeutlicht die Vielschichtigkeit von Benachteiligungen, weil sich ständig Verschiebungen, Neuzuordnungen und Veränderungen ergeben. Das Fließende dieser Kategorie ermöglicht zudem auch eine gewisse Freiheit über die (subjektive wie objektive) Zuordnung zu oder die Abgrenzung von einer Altersgruppe. Wie alle Differenzkategorien ist das Alter ein Produkt von sozialen Aushandlungsprozessen. Jedoch erscheint gerade die soziale Aushandlung des Alters durch den Doppelcharakter von Strukturkategorie und Prozess (van Dyk, 2015a, 2017) noch vielschichtiger, weil das stetige Fortschreiten des Alters eine ständige Neuzuordnung innerhalb der Kategorie erfordert. Die unterschiedlichen Aushandlungen von Alter als Kategorie und Prozess machen das Alter im Zusammenspiel mit anderen Differenzkategorien zu einem schwer greifbaren Faktor der Ungleichheit. Hier knüpft die Kernthese dieses Beitrags an: Wir argumentieren, dass die Räumlichkeit des Sozialen einen Zugang bietet, um die Dualität des Alters zu fassen und dass, darauf aufbauend, der Raum ein vielversprechender Ausgangspunkt für die analytische Erkundung der vielfachen Verflechtungen von Alter(n) mit anderen Differenzkategorien ist. Geographische Betrachtungen von Ungleichheit begreifen soziale Disparitäten als räumlich verortete, im und durch den Raum entstehende Erscheinungen (Rothfuß und Gerhard, 2014; Valentine, 2007). Differenzkategorien werden raumabhängig wahrgenommen und dargestellt, und so bestimmt sich gerade über den Raum mit welcher Konnotation die Kategorie Alter versehen und wie ein älterer Mensch wahrgenommen wird. Für eine Auseinandersetzung mit Alter und Ungleichheit ist das Mitdenken des Raums daher essentiell.

Mit diesem Beitrag wollen wir einen Impuls für die Aufarbeitung der erheblichen Forschungslücke setzen, die an der Schnittstelle zwischen ungleichheitssensibler Intersektionalitätsforschung und geographischer Altersforschung besteht. Bestehende Forschung zu Ungleichheit und Alter konzentriert sich auf einzelne Merkmale und rückt abhängig von theoretischer Verortung und Perspektive der Forschungsansätze unterschiedliche Aspekte von Ungleichheit in den Vordergrund. So geraten aus einer feministischen Perspektive vor allem die Benachteiligungen von älteren Frauen in den Blick (vgl. Calasanti und Slevin, 2006; Twigg, 2004; Sontag, 1972), marxistische Ansätze und die politische Ökonomie sehen in der sozialen Klasse den Ansatzpunkt für soziale Disparitäten im Alter (vgl. Estes, 1979; Townsend, 1981), Debatten aus der Migrationsforschung kritisieren eine Benachteiligung aufgrund von Ethnizität (vgl. Merrell et al., 2006; Tucci und Yildiz, 2012) und aus den Gender Studies wird auf Ungleichheiten für nicht-heterosexuelle Ältere hingewiesen (vgl. Cronin und King, 2010; Lottmann et al., 2016). Über diese einzelnen Aspekte hinaus bedarf es zudem einer Betrachtung der Verwobenheit von Differenzkategorien, um die komplexe Entstehung von Ungleichheiten zu verstehen. In der Intersektionalitätsforschung, die das Zusammenwirken von Differenzkategorien wie Ethnizität, soziale Klasse, Geschlecht, Sexualität, Alter, Religion oder körperlichen Einschränkungen betrachtet, ist das Alter bisher eine eher randständige Kategorie (Denninger und Schütze, 2017), die nur selten in die Analyse miteinbezogen wird und fast nie Ausgangskategorie der Forschung ist (Calasanti et al., 2006).

In der Humangeographie befasst sich die Teildisziplin der geographischen Altersforschung mit Alter und Gesellschaft (Skinner et al., 2015). Jedoch fehlt hier weitgehend der Blick auf Ungleichheiten und intersektionale Zusammenhänge. Durch die Altersblindheit der Intersektionalitätsforschung einerseits und das Vernachlässigen von Machtverhältnissen in der geographischen Altersforschung andererseits bleibt die Schnittstelle beider Disziplinen - das Zusammenspiel von Raum, Alter und Ungleichheit - unergründet. Im Folgenden stellen wir diese Wissenslücke anhand einer Literaturanalyse dar und zeigen, wie sich geographische und intersektionale Perspektiven auf Alter verbinden lassen. Hier fehlt es unserer Auffassung nach insbesondere an geeigneten methodologischen Zugängen, die es ermöglichen, das Alter in seiner Dualität als Kategorie und Prozess für intersektionale Analysen greifbar zu machen. Wir argumentieren, dass der Raum ein geeigneter Blickwinkel ist, durch den sich die komplexen Verwobenheiten des Alters mit anderen Differenzkategorien und daraus entstehende Privilegien und Ungleichheiten analysieren lassen.

Um unsere Argumentation zu entfalten, erläutern wir zunächst unser Verständnis der Begriffe „Ungleichheit“, ,Alter" und „Raum“: Unter Ungleichheit verstehen wir verschiedene Positionen, die Menschen im sozialen Machtgefüge einnehmen (Calasanti, 1996) und den daraus entstehenden Zugang zu Ressourcen. Der Ressourcenbegriff umfasst neben ökonomischen und politischen Ressourcen auch ,,[...] lebensnahe[n] Ressourcen, wie Gesundheit und soziale Netzwerke, in Form von Handlungskompetenz und Handlungsfähigkeit" (Clemens, 2008:28). Das Alter wird typischerweise abhängig von Diskurs und Bezugspunkt als biologisches, chronologisches, psychisches, soziales oder sichtbares Alter gefasst (van Dyk, 2015a). In diesem Artikel begreifen wir das Alter als ein sozial produziertes Merkmal, das - ähnlich wie die Prozesse des doing gender oder doing race - erst durch fortwährende soziale Herstellung und Aushandlungsprozesse seine Bedeutung erhält. Bei der Herstellung des höheren Alters wirken die unterschiedlichen Erscheinungsformen, wie eben chronologisch, biologisch, sichtbar oder sozial in verschiedenen Konstellationen zusammen. Für eine geographische Betrachtungsweise ist nach Harper (1997) der Körper ein besonders produktiver Anhaltspunkt, weil der alternde Körper zentral für die soziale Konstruktion von Alter und das Verdrängen älterer Menschen an bestimmte Orte ist.

Unser Raumverständnis orientiert sich an Henri Lefebvres Konzeption von Raum, die das Soziale und den Raum als untrennbar miteinander verwoben begreift (Lefebvre, 1991). 
In diesem Verständnis kann es nichts Soziales geben, das sich außerhalb des Raums abspielen würde: ,[...] räumliche Verhältnisse und Entwicklungen [sind] für Lefebvre sowohl Ausdruck als auch Voraussetzung für gesellschaftliche Verhältnisse“" (Ronnenberger und Vogelpohl, 2014:256). Die Produktion des Raums ist damit immer auch die Produktion des Sozialen. Konkret unterscheidet Lefebvre (1991) drei Dimensionen des Raums: den wahrgenommenen Raum, den konzipierten Raum und den gelebten Raum. Der wahrgenommene Raum (perceived space) ist der in seiner Materialität gelebte und genutzte Raum, der durch die alltägliche Nutzung - die räumliche Praxis - reproduziert wird. Der konzipierte Raum (conceived space) stellt die Dimension des Wissens, der Zeichen und der Codes dar, die über einen Raum bestehen und auf der Konzeptionsebene von Stadtplanern oder Politikern produziert werden, wie beispielsweise Raummodelle oder Karten. Der gelebte Raum (lived space) ist die imaginierte Dimension des Raums, welche die Erinnerungen an einen Raum, aber auch in die Zukunft gerichtet Visionen und Utopien für einen Raum beinhaltet. Aus den drei Dimensionen des Raums leitet Lefebvre (ebd.) drei Raumkonzepte ab: räumliche Praxis, die im wahrgenommenen Raum entsteht (1), die Repräsentation des Raums, die aus dem konzipierten Raum heraus produziert wird (2) und den repräsentativen Raum, der sich durch den gelebten Raum konstituiert (3).

Im Folgenden werden wir zunächst die Forschungslücke zwischen Intersektionalitätsforschung und geographischer Altersforschung aufzeigen und anschließend einen methodologischen Zugang zur Überbrückung dieser und der Beforschung des Zusammenspiels von Ungleichheit, Raum und Alter vorschlagen: den Raum als Forschungsperspektive.

\section{Intersektionale und geographische Perspektiven auf Alter(n)}

\subsection{Das Alter als vernachlässigte Kategorie der Intersektionalitätsforschung}

Die Entstehungsmechanismen für soziale Ungleichheiten sind Kernpunkte intersektionaler Forschung und Debatten. Trotz einer Vielfalt an Perspektiven auf die Hintergründe von Diskriminierungen wird das Alter nur selten mitgedacht. Für die überwältigende Mehrheit der Forschung ist das Alter der von Ungleichheit betroffenen Menschen unbedeutend und es werden irgendwie erwachsene, quasi alterslose Personen in die Forschung einbezogen (van Dyk, 2017). Als eigenständige Differenzkategorie wird Alter empirisch sowie konzeptionell kaum berücksichtigt und in der Intersektionalitätsforschung nicht angemessen anerkannt (Denninger und Schütze, 2017). So setzt sich die ,Alter(n)svergessenheit der Geschlechter- und Intersektionalitätsforschung“ (van Dyk, 2017:24) bereits über mehrere Jahrzehnte fort. Die Vernachlässigung von Alter in den Analysen sozialer Ungleichheit ist insofern erstaunlich, als sich die Intersektionalitätsforschung qua Definition mit sozialen Benachteiligungen beschäftigt und die Tatsache, dass das Alter die Lebenssituation verändert und zu Ungleichheiten führen kann, hinreichend bekannt ist (vgl. bspw. die Kontinuitätsthese, Kumulations- und Destrukturierungsthese und die These der Altersbedingtheit bei Clemens, 2008 und Mahne et al., 2017:22 oder in Bezug auf ökonomische Ungleichheiten bspw. Lejeune et al., 2017; Vogel und Motel-Klingenbiel, 2013). Beachtenswert sind in diesem Zusammenhang auch die Debatten aus der lebenslaufbezogenen Ungleichheitsforschung, die das Konzept des Cumulative Advantage/Disadvantage (CAD) mit dem Lebenslauf verbinden (vgl. bspw. Dannefer, 2003; Willson et al., 2007), sowie eine breite Diskussion über die Fortschreibung bestehender Ungleichheiten im Lebensverlauf (vgl. bspw. Kohli, 1988; Kohli et al., 2000; Künemund und Schröter, 2008; Scherger et al., 2011; Stoller und Gibson, 1994). Trotz dieser vielfältigen Anknüpfungspunkte für intersektionale Debatten spielt das Alter bislang kaum eine Rolle in der Intersektionalitätsforschung. Das Alter bleibt eine „Herausforderung für die Theorie der sozialen Ungleichheit" (Kohli, 1990:387), die von den Sozialwissenschaften bis heute nicht zufriedenstellend angenommen wurde.

In der frühen Intersektionalitätsforschung lässt sich die Schwerpunktsetzung auf Ethnizität, soziale Klasse und Geschlecht durch den Entstehungskontext Ende der 1980er Jahre in der US-amerikanischen Gesellschaft erklären. Die Juristin Kimberlé Crenshaw (1989) prägte das Bild des Unfalls auf einer Straßenkreuzung (intersection) zur Verdeutlichung der potentiellen Benachteiligungswirkung von Ethnizität und Geschlecht. Die Ursache von Diskriminierung könne, analog zur Straße aus der ein unfallverursachendes Fahrzeug kommt, nicht zweifelsfrei festgestellt werden, so Crenshaw (ebd.). Es kann aufgrund von Ethnizität, Geschlecht oder beiden Faktoren zu Diskriminierungen kommen. In der folgenden Begriffsgeschichte und der Verwendung im europäischen Kontext veränderten sich die Bezugspunkte. Abhängig von Schwerpunktsetzung und Erkenntnisinteresse werden die Unterscheidungsmerkmale modifiziert und ergänzt. So verweisen beispielsweise Lutz und Wenning (2001) auf 13 Linien der Differenz. Die Liste der möglichen Unterschiede und deren potentielle Auswirkung lässt sich prinzipiell unendlich erweitern. Es ist jedoch nicht das Ziel intersektionaler Herangehensweisen, eine vollständige Auflistung eventuell benachteiligender Attribute zu erstellen. Vielmehr geht es darum, die hinter den Ungleichheiten liegenden Machtgefüge zu verstehen.

Ob ein Zugang über Kategorien und Unterscheidungsmerkmale überhaupt weiterführend ist oder vielmehr bestehende Strukturen festschreibt, wird von unterschiedlichen Strömungen innerhalb der Intersektionalitätsforschung kontrovers diskutiert (vgl. McCall, 2005). Ebenso wird die Priorisierung bestimmter Differenzmerkmale aufgrund einer theoretischen Vorannahme kritisiert, weil damit der Blick auf alternative Einflussparameter verstellt werden kann (McMullin, 2000). Es wird aus gerichteten, durchaus auch normativ 
geprägten Perspektiven argumentiert, was die Wahrnehmung möglicher anderer Zusammenhänge erschwert. Bestimmte Unterscheidungsmerkmale sind in Diskurs und Theorie eingeübt, während auf andere Unterschiedlichkeiten (oder Gemeinsamkeiten) nur selten Bezug genommen wird. Calasanti (1996:148) stellt für Alter und Armut treffend fest: „Both African Americans and Mexican Americans have relatively high risks of poverty in retirement; if the importance of race/ethnicity is its relation to socioeconomic status, then why investigate these two groups separately? And why not just examine socioeconomic status?"“

Das Alter ist solch ein Parameter, der im Schatten der etablierten Kategorien steht und regelmäßig übersehen wird. Versuche aus feministischer Forschung und den Gender Studies, das Alter als erklärenden Faktor in intersektionale Analysen zu integrieren, stoßen außerhalb eines kleinen Diskurses auf wenig Resonanz. Die hier vorhandenen Überlegungen zu Ungleichheiten im Alter in der Intersektion mit Geschlecht (vgl. Calasanti et al., 2006; Calasanti und Slevin, 2006; Krekula, 2007; Sontag, 1972), mit Ethnizität und Geschlecht (vgl. Matthäi, 2005; Wray, 2003) oder Sexualität (vgl. Cronin und King, 2010; Denninger und Schütze, 2017; Lottmann et al., 2016; Schütze, 2017; Traunsteiner, 2018) werden vom Mainstream der Intersektionalitätsforschung kaum aufgegriffen. Mitunter wird das Alter unter der Kategorie „Körper“ zusammengefasst und steht gemeinsam mit körperlicher Einschränkung und Erscheinungsbild in einer diffus wirkenden Sammelkategorie (vgl. Winkler und Degele, 2010). Über den Körper lassen sich in Bezug auf Geschlecht, Ethnizität und soziale Klasse nicht mehr oder weniger verlässliche Aussagen treffen als über das Alter. Trotzdem wird der Trias Geschlecht, Ethnizität und soziale Klasse eine eigenständige Erklärungsmacht für Diskriminierung und Ungleichheit zugestanden - der Kategorie Alter hingegen nur sehr bedingt. Beispielhaft zeigt sich dies auch in der sprachlichen Repräsentation von Diskriminierungen: Während Rassismus und Sexismus als Begriffe etabliert sind, bleibt die Debatte um ageism (Butler, 1969), insbesondere im deutschsprachigen Raum, eine wenig beachtete Randdiskussion (van Dyk, 2015a). Die Fokussierung auf bestimmte Kategorien prägt die bestehenden Beiträge zu Alter und Ungleichheit. Ein Großteil der Forschung, der sich mit Alter im Zusammenhang mit anderen Differenzmerkmalen auseinandersetzt, betrachtet explizit eine soziale Gruppe in der Alterung (vgl. bspw. für Ethnizität Baykara-Krumme et al., 2012; Rohstock, 2014 oder für Menschen mit Behinderung Havemann und Stöppler, 2009). Der Forschungsansatz ist dabei nicht unbedingt intersektional, vielmehr steht das Alter(n) einer als ,anders“ verstandenen Gruppe im Mittelpunkt (Calasanti, 1996). Intersektionale Ansätze, die über eine Auseinandersetzung mit der Alterung einzelner sozialer Gruppen hinausgehen und das Alter selbst konzeptionell durchdenken, sind im Vergleich zu anderen Kategorien der Differenz kaum vertreten.
Ein möglicher Grund für diese große Forschungslücke, so vermuten wir, liegt in der fehlenden Auseinandersetzung mit der binären Konstruktion von Alter. Die Dekonstruktion binären Denkens, das ein „Normales“ und ein „Anderes" in sich trägt, haben die Gender Studies und die Postcolonial Studies für die Gegensatzpaare „Mann - Frau“ bzw. „schwarz - weiß“ längst vorgemacht und beispielsweise gezeigt, dass die Kategorie „Geschlecht“ sozial konstruiert ist und sich auch das Weiß-Sein mit der Kategorie race beschreiben lässt (Hopkins und Pain, 2007). Eine Dekonstruktion des binären Begriffspaars ,Jung - Alt“ wurde bislang kaum diskutiert (vgl. kritisch McMullin, 1995; van Dyk, 2017). „Jung sein“ ist die positive Norm gegenüber der das „Alt sein“ konstruiert wird, wobei „Jung“ stets auf die „Nicht-Alten“ der mittleren Lebensjahre verweist und nicht etwa auf Kinder und Jugendliche (van Dyk, 2015b). Die Anerkennung, dass jede Lebensstufe ein Alter hat und nicht nur die beiden Extreme Kindheit/Jugend und höheres Alter, taucht in den Debatten kaum auf (Hopkins und Pain, 2007) und so bleiben die mittleren Lebensjahre gewissermaßen frei von jedem Alter. Das Bild des erfolgreichen Alter(n)s spiegelt letztlich den Lebensstil dieses, vermeintlich altersfreien, Lebensabschnitts wider (Calasanti et al., 2006). Populäre Narrative wie die Figur der ,,jungen Alten“ oder das Schlagwort des ,aktiven Alter(n)s“ verschleiern die binäre Konstruktion, indem die Eigenschaften des mittleren Alters zu einer Maxime für alle Erwachsenen erhoben werden. Das höhere Alter wird zu einer Lebensphase gemacht, die durch Disziplin, Aktivität und Teilhabe vermieden werden kann (van Dyk, 2015b, 2017). Prägnant formuliert: „Getting old is a sign of giving in“ (Mowl et al., 2000:191).

\subsection{Ungleichheit in der geographischen Altersforschung?}

Ebenso wie Alter in der Intersektionalitätsforschung nur selten beforscht wird, spielen in der geographischen Altersforschung Ungleichheit und Intersektionalität keine nennenswerte Rolle. Die thematische Breite der geographischen Altersforschung ist zwar beachtlich (vgl. Skinner et al., 2015:778), allerdings bleibt eine Auseinandersetzung mit den in der Kategorie Alter enthaltenen Machtstrukturen und Ungleichheiten unterrepräsentiert.

Klassische Themen der geographischen Altersforschung sind die räumliche Verteilung älterer Menschen, die Bereitstellung von Infrastruktur und Dienstleistungen, sowie Gesundheit, Wohnen und das direkte Lebensumfeld (Andrews et al., 2009; Helbrecht und Geilenkeuser, 2012). Die Empirie basierte, anwendungsorientierte Herangehensweise der frühen Gerontologie (van Dyk, 2015a) setzte sich zunächst auch in der Auseinandersetzung mit Alter aus einer räumlichen Perspektive fort. Der Forschungsgegenstand der ,Alten" wurde, wenn überhaupt, durch eine chronologische Abgrenzung definiert. Diversität und Unterschiede innerhalb der Gruppe der „Älteren“ spielten für die positivistisch ausge- 
richtete Forschung nur eine untergeordnete Rolle (Harper und Laws, 1995). Die deutschsprachige Altersgeographie ist hier keine Ausnahme. Sie konzentriert sich bis heute vor allem auf anwendungsorientierte Fragen im Feld des altersgerechten Wohnens (vgl. Oswald et al., 2013; Schneider-Sliwa, 2004) oder der altersfreundlichen Stadtgestaltung (vgl. Franz und Vaskovics, 1982; Nowossadek und Mahne, 2017). Eine konzeptionelle Auseinandersetzung mit dem Faktor Alter konnte sich in der deutschen Geographie nicht etablieren, und es fehlt die Verbindung zu kritischen Strömungen in der Geographie wie beispielsweise der Neuen Kulturgeographie.

Für die internationale geographische Forschung kann diese Diagnose heute nicht mehr uneingeschränkt beibehalten werden. Feministische, poststrukturalistische und postmoderne Denkansätze wirken auch in der geographischen Altersforschung und befördern Debatten um das subjektive Erleben des Alters, das Alltagsleben älterer Menschen und die Rolle des alternden Körpers. Trotz dieser neuen Forschungsfelder werden kritische, konzeptionell orientierte Ansätze in der geographischen Altersforschung noch immer weniger behandelt als anwendungsorientierte Fragestellungen. Die Repräsentation oder die Performanz des Alters und der Verlauf von Altersprozessen werden kaum untersucht (Skinner et al., 2015). Um Ungleichheiten im Alter aus einer intersektionalen Perspektive zu verstehen, sind es aber genau diese Fragen um Repräsentation, Konstruktion und Performanz des Alter(n)s, die einer Betrachtung bedürfen. Auch wenn Ungleichheit nicht explizit als Thema der geographischen Altersforschung formuliert wird, sind in einzelnen Beiträgen wichtige Überlegungen zur Relationalität von Alter sowie zu Alter und Differenz enthalten (vgl. Henery, 2011; Hopkins und Pain, 2007; Pain et al., 2000; Mowl et al., 2000; Schwanen et al., 2012). Es gilt, diese Denkansätze aufzugreifen und ihr Potenzial für eine ungleichheitssensible, intersektional ausgerichtete Geographie des Alter(n)s explizit hervorzuheben und weiterzuentwickeln. Zwei Erkenntnisse aus der bestehenden Forschung halten wir für besonders instruktiv:

Erstens ist für eine intersektional ausgerichtete $\mathrm{Al}$ ter(n)sgeographie die Einsicht fundamental, dass Alter eine relationale Größe ist. Das Alter entsteht erst in Relation zu anderen gegenüber denen jemand als älter oder jünger erscheint. Wenn das Alter in seiner Relationalität als sozial produziert - statt als feststehende Größe - verstanden wird, gewinnen räumliche Faktoren an Einfluss (Hopkins und Pain, 2007). Die institutionalisierte Alterssegregation an öffentlichen Orten wie Kindergärten, Schulen, Arbeitsstellen oder Seniorenfreizeiteinrichtungen zeigt deutlich, dass die altersbezogene Stratifizierung der Gesellschaft auch über den Raum funktioniert und gerade dadurch relationale $\mathrm{Ab}$ grenzungsverhältnisse der Altersgruppen zueinander und damit verbundene, altersspezifische Verhaltensnormen und Erwartungen an bestimmte Altersgruppen produziert werden (Hagestad und Uhlenberg, 2005). Über die Analyse der KoKonstruktion von Raum, Identität und Alter können Unterschiedlichkeiten, Widersprüche und Ungleichheiten in der
Repräsentation und Wahrnehmung von Alter aufgezeigt werden. Es wird deutlich, dass die Kategorie Alter nicht allein eine chronologische Größe ist, sondern vielmehr als eine kontextabhängig konstruierte, soziale Zuschreibung wirksam wird. Damit rückt die Komplexität der Verwobenheit von verschiedenen Differenzkategorien und den damit verbundenen Privilegien und Benachteiligungen in den Vordergrund: „A primary question is always who else older, middle-age or younger people are“ (Hopkins und Pain, 2007:290, Hervorhebung im Original). Das relationale Verständnis von Alter wird also nicht nur in Beziehung zu Menschen anderer Altersgruppen wirksam, sondern auch im Zusammenhang mit anderen Differenzmerkmalen. Zwischen Menschen, die chronologisch gesehen in einem Alter sind, können Räume als Abgrenzungsmechanismen wirken. Pain et al. (2000) zeigen am Beispiel von Freizeiteinrichtungen in England, wie Menschen sich gezielt in Räumen bewegen, um sich in Relation zu einer anderen Gruppe als „,nicht ganz so alt“ abzugrenzen, so beispielsweise Männer zu Frauen, Angehörige der Mittelschicht zur Arbeiterklasse oder gesunde zu körperlich eingeschränkten Menschen. Dabei werden Räume strategisch genutzt, um altersfeindlichen Diskursen auszuweichen und das Stigma ,alt" auf andere zu verschieben. Auf welche Weise diese Strategien genutzt werden können, ist eng mit intersektionalen Unterschiedlichkeiten wie sozialer Klasse, körperlicher Verfassung und Geschlecht verknüpft (ebd.).

Ein zweiter Zugang, über den Alter in der Geographie als relational und intersektional verstanden werden kann, ist die Körperlichkeit des Alter(n)s. Um sich von einer biologistischen Sicht auf den Körper abzugrenzen, untersuchte die Sozialgerontologie lange vorwiegend gesellschaftliche und soziale Aspekte des Alter(n)s. Dies verändert sich gegenwärtig vor allem durch Beiträge aus der feministischen Gerontologie (vgl. Krekula, 2007; Höppner, 2017; Twigg, 2004; Slevin, 2006) und aus den embodied geographies der geographischen Altersforschung (Skinner et al., 2015). In den Körper schreiben sich soziokulturelle und physisch-biologische Attribute gleichsam ein, und die Diversität im Altersprozess tritt konkret und sichtbar zutage (Schwanen et al., 2012). Gleichzeitig ist der Körper auch (vermeintlicher) Anhaltspunkt für die Einordnung in Differenzkategorien wie Geschlecht oder Ethnizität. Ein geographischer Blick, der den alternden Körper in seine Umgebung einbettet und den Körper tatsächlich in seiner Materialität im Raum denkt, lenkt die Aufmerksamkeit auf die biologische Komponente des Körpers. Am alternden Körper lässt sich ablesen, dass das Alter Kategorie und Prozess zugleich ist. Es sind zutiefst körperliche Komponenten wie graues Haar, der Gang, Falten, die Haltung oder die Bewegungssicherheit, die als Anhaltspunkte für die Einordnung innerhalb der Differenzkategorie Alter dienen. Gleichzeitig zeigt sich am alternden Körper, dass die Alterung ein Prozess ist. Unabhängig vom chronologischen Alter verändert sich der Körper bei allen Menschen unterschiedlich. Ungleichheiten in der körperlichen Verfassung im Alter können in intersektionalen Überschneidun- 
gen auftreten (vgl. Klaus und Baykara-Krumme, 2017), aber ebenso intersektionale Hierarchien durchkreuzen (Vincent, 1995). Die Einbettung des Körpers in den räumlichen Kontext macht sichtbar, dass Raum und Körper in einer engen Wechselwirkung miteinander stehen (Schwanen et al., 2012; Mowl et al., 2000). Wie ein Körper gesehen wird und welche Fähigkeiten ihm zugeschrieben oder abgesprochen werden, wird auch durch die soziale Konnotation eines Raums bestimmt (vgl. für die verjüngende Wirkung von Seniorensiedlungen McHugh, 2007).

\section{Der Raum als Forschungsperspektive auf Ungleichheit und Alter}

Unsere Analyse des Wissensstands in der Intersektionalitätsforschung zur Kategorie Alter und in der Altersgeographie zu Ungleichheit zeigt, dass das Zusammenspiel von Ungleichheit, Raum und Alter weitgehend unerforscht ist. Dass Identitätsbildung und die Herstellung von Hegemonie und Dominanz in Wechselwirkung mit Räumen geschieht, wurde in der Humangeographie überzeugend dargelegt (vgl. z.B. Valentine, 2007). Explizit für das Alter wird die Bedeutung des Raums bei Hopkins und Pain (2007:287f) formuliert: „,This shift from seeing age and lifecourse as socially constructed variables rather than independent variables means that space and place gain significance. People have different access to and experiences of places on the grounds of their age, and spaces associated with certain age groups influence who uses them and how. Further, people actively create and resist particular age identities through their use of space and place (see Pain et al. 2001; Valentine 2004).“ Allerdings wird hier das Alter rein als Strukturkategorie gefasst und somit als feste Größe zu einem gegebenen Zeitpunkt betrachtet. Damit bleibt die zweite Facette des Alters, der Altersprozess, ausgeklammert.

Im Folgenden gehen wir daher der Frage nach, wie die Aushandlung von Alter vor dem Hintergrund der Anerkennung des Doppelcharakters als Kategorie und Prozess - und darauf aufbauend das Zusammenspiel mit anderen intersektionalen Kategorien - beforscht werden kann. Dabei sehen wir den Raum als methodologischen Zugang, der es erlaubt, die Dualität von Alter und Altern zu fassen und im $\mathrm{Zu}$ sammenspiel mit anderen Differenzkategorien zu analysieren. Wir folgen dem Raumverständnis von Lefebvre (1991) und verstehen Raum und soziale Welt als in einem ständigen Wechselspiel begriffen. Diese Herangehensweise erlaubt es, den Raum als Analyseinstrument für das Soziale zu nutzen: „From the point of view of knowing (connaissance) social space works (along with its concept) as a tool for the analysis of society. To accept this much is at once to eliminate the simplistic model of a one-to-one or punctual correspondence between social actions and social locations, between spatial functions and spatial forms." (Lefebvre, ebd.: 34) Mit dieser Konzeption der Räumlichkeit des Sozialen blicken wir im Folgenden auf die Aushandlung von Alter als Kategorie und Prozess. Der Raum als methodologisches Prisma eröffnet einen gerichteten Blick auf die Interrelationen zwischen Alter und Ungleichheiten und bietet einen Ausgangspunkt, um den intersektionalen Verschränkungen nachzugehen.

\subsection{Alter als Prozess: Flexible Selbstzuschreibung und Deutungshoheit}

Blickt man auf das Alter als den Prozess des Alterns, so ist es geradezu das Wesen dieses Prozesses, dass jeder Mensch verschiedene Altersstufen durchläuft und dabei unterschiedliche Positionen im gesellschaftlichen Machtgefüge einnimmt. In dieser Hinsicht bedarf die Aushandlung der Kategorie Alter einer besonderen Betrachtung. Der Aufbau von Gegensätzlichkeiten nach dem Identifikations- und Abgrenzungsschema „Wir“ und ,die Anderen“ entlang von Merkmalen wie Geschlecht, Sexualität, Ethnizität oder Religion ist für das Alter so nicht durchzuhalten. Auf der diskursiven Ebene, die sich in ihrer Gruppenbildung und Zuweisung von Eigenschaften an Alter als Strukturkategorie zu orientieren scheint, ähnelt die Gegenüberstellung von Jung und Alt anderen binären Konstruktionen. Die individuelle Verarbeitung der Zugehörigkeit zu einer Altersgruppe erscheint dagegen komplizierter, weil Zuordnungen und Selbstpositionierungen im Lauf des Lebens ständig neu auftauchen und verschwinden (van Dyk, 2015b). Ohne Zweifel gibt es auch Menschen, die zwischen anderen Kategorien stehen und sich zwischen ihnen bewegen. Für das Altern als Prozess ist das Durchlaufen verschiedener Altersstufen aber gerade der wesentliche Kern. Man erlernt es gewissermaßen, die Zugehörigkeit zu Alterskategorien zu wechseln und das Alter als bewegliche Kategorie zu behandeln. Diese Flexibilität wirkt nun nicht nur auf größere zeitliche Zusammenhänge wie den Lebensverlauf, sondern auch in der Gegenwart. Als laufender Prozess erlaubt das Alter offenbar einen flexiblen Umgang mit der $\mathrm{Zu}$ ordnung und Abgrenzung vom Attribut ,höheres/hohes Alter". Es liegt in der Hoheit der Menschen selbst, in welchem Kontext sie sich subjektiv mit der Gruppe der Älteren identifizieren und wann nicht. Um diese subjektive Einordnung nach außen zu kommunizieren, werden Räume strategisch genutzt. Selbstverständlich entstehen Selbstzuschreibungen und die Verortung in bestimmten Räumen nicht ausschließlich aus strategischem Kalkül heraus mit dem Ziel, sich einer Altersgruppe zuzuordnen oder von ihr abzugrenzen, sondern können ebenso aus Gewohnheiten, Gruppenzugehörigkeiten oder pragmatischen Gründen erwachsen. Im Folgenden konzentriert sich der Beitrag jedoch auf die strategische Nutzung von Räumen, da diese am deutlichsten veranschaulicht, welche Wirkungsmacht das Wechselspiel von Raum und Sozialem entfaltet. Anhand der drei Raumdimensionen von Lefebvre - der wahrgenommene, der konzipierte und der gelebte Raum - lässt sich die strategische Nutzung systematisch durchdenken. 
In den Dimensionen des konzipierten und des gelebten Raums liegt begründet, dass bestimmte Orte überhaupt mit einer Altersgruppe assoziiert werden. Räume und Orte werden von Planung und Politik für eine bestimmte Zielgruppe geplant und gedacht. So bedarf beispielsweise die Altersausrichtung von Altersheimen, Schulen oder Diskotheken keiner dezidierten Erklärung. Die Selbstverständlichkeit, mit der Räume nur einer Altersgruppe zugeordnet werden, wird über den gelebten Raum stabilisiert. Durch Erinnerungen an einen Raum wird das Bild einer Örtlichkeit geprägt, sodass die Präsenz einer anderen Altersgruppe in diesem Raum seltsam anmutet. Auf diese klare Zuordnung von Räumen zu einer bestimmten Altersgruppe trifft nun der wahrgenommene Raum und die räumliche Praxis. Hier zeigt sich die Handlungsmacht der Subjekte über die Einordnung in eine bestimmte Altersgruppe. Die räumliche Praxis der Akteure, also wo und auf welche Weise sich jemand positioniert, ist ausschlaggebend dafür, wie das Alter ausgehandelt wird. Akteure können die Verknüpfung eines Raumes mit einer Altersgruppe oder einem bestimmten Bild des Alters nutzen, um ihre subjektive Einordnung in die Gruppe der Älteren oder Jüngeren (oder die Abgrenzung davon) nach außen zu tragen. An welchem Ort sich jemand positioniert, kommuniziert damit die Zuordnung zu einer Gruppe und zu einem Altersbild. So wird beispielsweise eine Person, die sich in einer Seniorenvertretung politisch engagiert, aktiver - und damit jünger - wirken als jemand, der regelmäßig zum nachbarschaftlichen Seniorentreff geht. Der Ort ist hier entscheidend für die Aushandlung des Alters. Gleichzeitig ist aber auch die Art und Weise bedeutsam, wie sich jemand in einem Raum positioniert. Eine ältere Person, die Besuchsdienste im Altersheim übernimmt oder den nachbarschaftlichen Seniorentreff für andere Ältere organisiert, ist im Rahmen dieser Orte das aktive - und damit jüngere - Element und trägt dies auch nach außen.

Für eine Auseinandersetzung mit Alter und sozialer Ungleichheit ist die Räumlichkeit als Prisma ein sinnvoller $\mathrm{Zu}$ gang, um zu untersuchen, wovon es abhängig ist, wer welchen Raum strategisch nutzen kann, um eine subjektive Alterszuschreibung nach außen zu tragen. Die verfügbaren Ressourcen einer Person, wie beispielsweise die finanzielle Lage, die körperliche Verfassung oder soziale Netzwerke bestimmen, wer sich wo und auf welche Weise im Raum positionieren kann und damit die Chance auf die Darstellung des subjektiven Altersbilds nach außen hat. Außerdem lassen sich über die strategische Nutzung von Räumen verschiedene Konzeptionen des Alters und Altersbilder erfassen. Welcher Raum aus welchem Grund von einer Person gewählt wird, um das Altersbild nach außen zu tragen, gibt wichtige Hinweise auf die Verarbeitung des Altersprozesses im Wechselspiel mit unterschiedlichen Differenzkategorien. Eine Analyse der Darstellung des Altersbilds nach Außen erlaubt es, die subjektive Wahrnehmung des Alters zu ergründen. Über eine solche Herangehensweise lassen sich auch Diskurse über das Alter aufnehmen, die jenseits der großen Erzählungen vom ,,aktiven Alter“ oder der Pflegebedürftigkeit bestehen. Dass die Lebensphase Alter durchaus als würdevoll und reich an Erinnerungen und Erfahrungen, ja sogar als wünschenswert, erlebt werden kann, kann durch die Beschäftigung mit konkreten Orten sichtbar und in den großen Diskursen hörbar gemacht werden. Der Raum erweist sich hier als vielversprechender methodologischer Ausgangspunkt für eine Analyse des Zusammenspiels von Alter(n) mit anderen Kategorien und daraus entstehenden Ungleichheiten, denn er ermöglicht sowohl den Blick aus der Makroperspektive - Darstellung des Alters in Relation zu dominanten Diskursen - als auch einen Blick aus der Mikroperspektive - die persönliche Verarbeitung des Altersprozesses.

\subsection{Alter als Strukturkategorie: Die Produktion durch den Raum}

Im Gegensatz zum flexiblen Umgang mit der Selbstzuordnung zur Kategorie ,,alt“ aus der Prozessperspektive verläuft die Einordnung in die Strukturkategorie Alter restriktiver. Über klar benennbare Faktoren wie das chronologische Alter, das Erscheinungsbild, die körperliche Verfasstheit oder soziale Rollen und Zäsuren im Leben, wie beispielsweise den Eintritt in das Rentenalter oder die Annahme der Großelternrolle, werden Menschen gesellschaftlich mit dem Attribut ,alt“ versehen. Dabei werden der Gruppe der Älteren beschreibbare Eigenschaften und (abnehmende) Kapazitäten zugeordnet. In dieser Hinsicht wirkt das Alter als klar definierte soziale Einordnungskategorie, die deutliche Abgrenzungen schafft. Mit der Herangehensweise, den Raum als Analyseinstrument für soziale Prozesse einzusetzen, kann die Herstellung der Kategorie Alter für sich und in Relation zu anderen Kategorien über Räume sichtbar gemacht werden. Dies zeigen wir im Folgenden anhand dreier Wohnformen für ältere Menschen: das Altersheim, eigenständiges Wohnen im Modellprojekt Pflege@Quartier und das im Entstehen begriffene Berliner Wohnquartier future living. Die drei Räume bilden ein Kontinuum von einer restriktiven Aushandlung des Alter(n)s bis zur völligen Offenheit ab. Erneut nutzen wir Lefebvres Raumdimensionen zur systematischen Darstellung unserer Überlegungen, um zu zeigen, welchen Spielraum die einzelne Räumlichkeit für die Aushandlung von Alter eröffnet und inwiefern andere Kategorien bei der Aushandlung einen Platz haben.

\subsubsection{Restriktive Aushandlung: Das Altersheim}

Das Altersheim ist ein Raum, der nur wenig Spielraum für die Aushandlung von Alter lässt. Die Planung eines Raums als Altersheim - der konzipierte Raum - stellt das Altern des Körpers und die Abnahme der körperlichen und geistigen Fähigkeiten in den Mittelpunkt. Sichtbar wird das in der materiellen Ausstattung des Raums mit beispielsweise Notklingeln, Haltegriffen in Sanitäranlagen oder an der unübersehbaren Präsenz von Rollstühlen und Rollatoren. Das vorherrschende Altersbild ist ein defizitorientiertes, das auf Pfle- 
gebedürftigkeit und Abhängigkeit ausgerichtet ist. Der konzipierte Raum „Altersheim“ richtet sich an das hohe Alter und steht symbolisch für das Ende des Lebens. Nur selten wird jemand noch einmal aus dem Altersheim ausziehen. Die Planung von Orten, die Menschen nur auf Basis des Alters von der Gesellschaft segregieren, weist auf die Verdrängung des Alters aus der Gesellschaft hin (Vincent, 1995). Im Altersheim als konzipiertem Raum ist kein Platz für die Aushandlung von Alter. Das beeinflusst auch die Raumdimensionen des gelebten und des wahrgenommenen Raums. Wie kaum ein anderer Ort steht das Altersheim für Leiden, Tod und zunehmend auch für Vernachlässigung und (schlechte) Pflege (Milligan, 2009). Die Dimension des gelebten Raums, die sich aus Erinnerungen an und Assoziationen mit einem Ort speist, unterscheidet sich in den Annahmen nicht nennenswert von denen des konzipierten Raums. Daraus resultiert eine regelrechte Furcht vor Altersheimen, die einen Umzug ins Pflegeheim für die Wenigsten wünschenswert macht (Oswald, 2013). Auch hier lässt der Raum also keine Perspektive für eine alternative Aushandlung des Alters. Drittens produziert die räumliche Praxis im wahrgenommenen Raum das defizitorientierte Altersbild. Die immer gleichen Routinen im Pflegealltag, die nur wenig Rücksicht auf individuelle Bedürfnisse der Menschen nehmen (können), reproduzieren und verstärken die Abhängigkeit und Passivität der Heimbewohner und damit die Konzeption von Alter als bedauernswertes Ende des Lebens.

Im Altersheim wirken andere Strukturkategorien kaum auf die Aushandlung des höheren Alters. Zwar werden jüngst Diskussionen über kultur- und biographieorientierte Pflege geführt (z.B. Khan-Zvornicanin, 2016 für interkulturelle Altenpflege; Linschoten et al., 2016 für die Pflegebedürfnisse nicht-heterosexueller Älterer), allerdings überstrahlt der fragile Körper die Wirkmacht anderer Strukturkategorien. Fixpunkt bleibt die Frage nach der Sicherstellung von Pflege und der „Versorgung“ der alten Person. Als Raum produziert das Altersheim die Strukturkategorie Alter in einer Weise, die weder Spielraum für alternative Aushandlungen lässt noch eine nennenswerte Wirkmacht anderer Differenzmerkmale in den Debatten erlaubt.

\subsubsection{Aushandlung von ,jungem“ und „hohem“ Alter: "Pflege@Quartier"}

Etwas mehr Platz für die Aushandlung des Alters bietet der Raum „Pflege@ Quartier“. Als Modellprojekt der städtischen Wohnungsbaugesellschaft GESOBAU (Berlin) und der AOK Nordost soll diese Wohnform ,die Erhaltung der Selbstständigkeit im häuslichen Wohnbereich" ermöglichen (GESOBAU, 2018). Angesiedelt in den herkömmlichen Wohnungen der städtischen Wohnungsbaugesellschaft erlaubt diese Wohnform einerseits ein Wohnen ohne jede Hilfe von außen. Andererseits verfügt die Wohnung über technische Unterstützungsleistungen wie Sturzerkennung, Inaktivitätsmelder oder Orientierungslichter zum Sanitärbereich, sollten körperliche Beeinträchtigungen zunehmen (ebd). Als konzipierter Raum soll es diese Wohnform möglich machen, auch im Alter in der gewohnten Umgebung zu bleiben. Dabei wird der Raum durch die technische Ausstattung als innovativ und zukunftsorientiert kommuniziert. Solange man nicht auf die Unterstützung angewiesen ist und keines der technischen Hilfsmittel nutzt, kann das Alter in dieser Wohnung frei gestaltet werden. Gleichzeit ist aber auch hier die Körperlichkeit des Alters der Referenzpunkt, an dem sich die technische Ausstattung orientiert. In der Materialität des Raums demarkiert die Abnahme der körperlichen Fähigkeiten die Grenze zwischen jungem, aktivem Alter und der Hochaltrigkeit. Die räumliche Ausstattung schreibt den Weg in das Alter als Abhängigkeit bereits vor.

Für die Aushandlung der Strukturkategorie „Alter“ ist insbesondere Lefebvres Dimension des wahrgenommenen Raums und das Raumkonzept der räumlichen Praxis interessant. Der Übergang zwischen aktivem Alter und dem Alter als Lebensphase mit mehr Unterstützungsbedarf ist meist fließend. Dadurch, dass „Pflege@Quartier“ es ermöglicht, auch mit größeren Einschränkungen in einer Wohnung zu bleiben, die bereits vorher mit Hilfsmitteln ausgestattet ist, gibt es keinen bestimmbaren Wendepunkt, der den Eintritt in das Alter als Lebensphase der Abhängigkeit markieren würde. Der Gestaltungsspielraum des Alters, den Bewohnerinnen und Bewohner in diesem Raum in ihrer räumlichen Praxis verhandeln, eröffnet eine gewisse Freiheit für die Aushandlung dessen, was höheres Alter bedeutet und welche Eigenschaften der Gruppe der Älteren zugeschrieben werden, die als Bewohnerinnen und Bewohner von „Pflege@Quartier“ sichtbar werden. So ist beispielsweise durch die innovative technische Ausstattung auch eine gewisse Aufgeschlossenheit gegenüber Technik und Medien ein Teil dessen, was Alt sein im Raum „Pflege@ Quartier“ bedeutet.

In Hinblick auf Alter als Kategorie der Ungleichheit lieBe sich entlang dieses Raums analysieren, inwiefern Differenzkategorien (z.B. Geschlecht, Bildungshintergrund) darauf wirken, wer das Angebot in Anspruch nimmt und wie Alter davon abhängig in dieser Wohnform verhandelt wird. Im konkreten Beispiel ist „Pflege@Quartier“ in der Großwohnsiedlung eines städtischen Wohnungsbauunternehmens am nördlichen Stadtrand Berlins verortet. Es wäre zu untersuchen, welche Motivationen dem Einzug in eine solche Wohnung zugrunde liegen und unter welchen Umständen die innovative technische Gestaltung der Anreiz ist, den Wohnort dorthin zu verlagern. Ob das Alter ein Faktor sein könnte, der Segregation auf Basis anderer Differenzkategorien entgegenwirkt, müsste am konkreten Beispiel geklärt werden. Ebenso ließe sich intersektional denken, welches Altersbild dem Wohnen in einer Wohnung mit dieser technischen AltenhilfeAusstattung zugrunde liegt. Im gelebten Raum der Erinnerungen, Assoziationen und Visionen könnte ein Raum wie „Pflege@Quartier“ auch mit Diskursen um die Technisierung der Pflege, beispielsweise durch Pflegeroboter, in Verbindung gebracht werden. Für wen „Pflege@ Quartier“ trotz- 


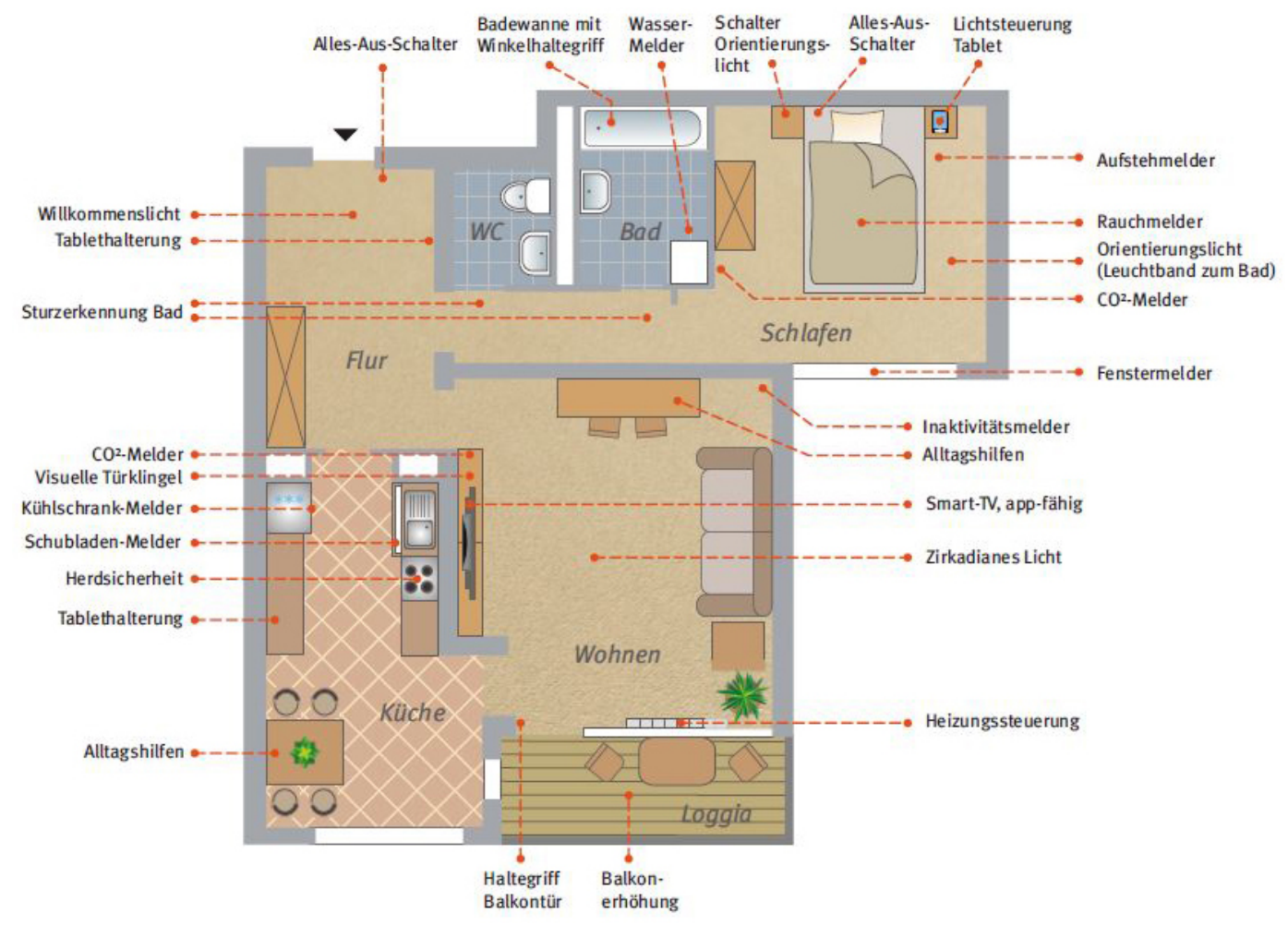

Abb. 1. Karte der Musterwohnung Pflege@Quartier. GESOBAU AG, Musterwohnung Pflege@Quartier. Online aufrufbar: https://www. gesobau.de/mieterservice/wohnkonzepte/pflegequartier.html (letzter Zugriff: 11 September 2018).

dem - oder gerade deshalb - ein attraktiver Wohnort sein kann, ist in der persönlichen Vorstellung der Lebensphase Alter begründet. Wie das Alter subjektiv ausgehandelt wird, lässt sich nicht unabhängig von anderen strukturellen Kategorien wie Geschlecht, Ethnizität oder sozialer Klasse denken. Der Raum „Pflege@Quartier“ ist ein fruchtbarer Ausgangspunkt, um das Zusammenspiel der unterschiedlichen Faktoren für die Aushandlung zu ergründen.

\subsubsection{Die Analyse des Aushandlungsprozesses von Beginn an: „future living" in Berlin-Adlershof}

Der dritte Raum, über den wir darlegen möchten, wie der Raum als Blickwinkel auf die Produktion von Alter als Strukturkategorie genutzt werden kann, ist das neuentstehende Wohnungsbauprojekt „future living“ in Berlin-Adlershof. Als konzipierter Raum ist future living nicht explizit auf eine Altersgruppe ausgerichtet, vielmehr sollen „,im Quartier Studenten, Singles, Familien und Senioren ein Zuhause finden.“ (GSW, 2018) Allerdings suggerieren Sätze wie „Moderne Technologien sowie bedarfsgerechte Dienstleistungen ermöglichen in allen Lebensphasen ein selbstbestimmtes Leben in der eigenen Wohnung auf einem komfortablen Ausstattungsniveau.“ (ebd.), dass sich das neue Quartier gezielt an ältere Menschen richtet. Der konzipierte Raum des future living ist gekennzeichnet von dem Wunsch für unterschiedli-

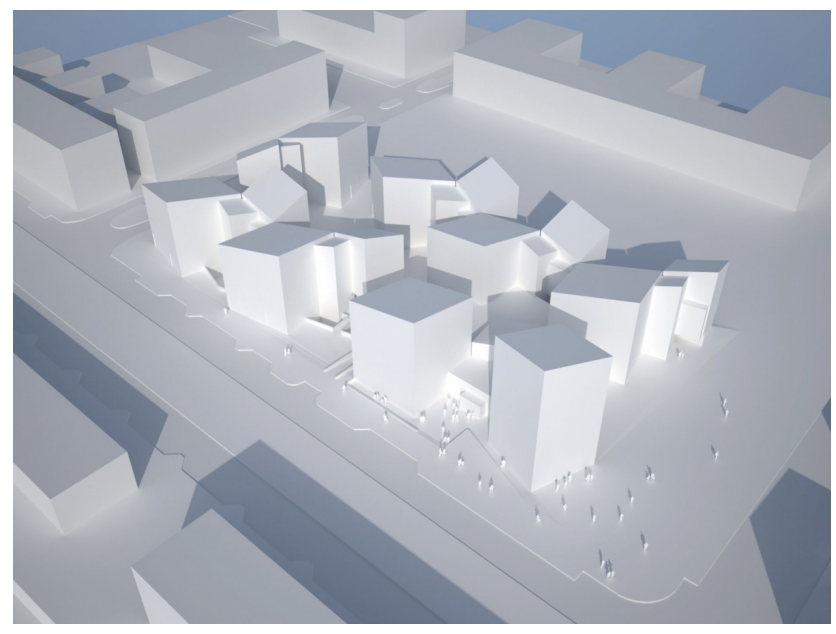

Abb. 2. Architekturmodell future living. GSW Sigmaringen mbH. Online aufrufbar: https://future-living-berlin.com/img/2017/ 07/future-living-berlin-3d-gebaeudeanordnung.jpg (letzter Zugriff: 11 September 2018).

che Generationen attraktiv zu sein. Die Planung barrierefreier Wohneinheiten, großzügiger Grünflächen zwischen den Gebäuden sowie die Bildsprache über das Bauprojekt weisen drauf hin (vgl. GSW, 2018). Für unser Interesse an der Aushandlung von Alter als Kategorie und dem Zusammen- 
spiel des Alters mit anderen Strukturkategorien ist der Raum future living besonders reich an Möglichkeiten zur Analyse. Der Raum ist im Entstehen, und bislang ungenutzt. Damit ist der wahrgenommene Raum noch unverbraucht und im Werden begriffen. Die Aushandlung des höheren Alters in diesem Raum ist offen. Der Raum future living bietet damit ein Prisma auf die sozialen Prozesse der Aushandlung unterschiedlicher Alterskategorien. Über räumliche Praxis, das Bewohnen und Aneignen des Raums von Menschen unterschiedlicher Altersgruppen, kann für den Ort neu verhandelt werden, was Alter als Strukturkategorie bedeutet. Die räumliche Praxis der Bewohnerinnen und Bewohner bestimmt, ob der Raum höheres Alter als Kategorie der Selbstidentifikation akzentuiert, wie es beispielweise Seniorensiedlungen in den Vereinigten Staaten tun (vgl. McHugh, 2007) oder ob der Anspruch, ein Ort für verschiedene Generationen zu sein, gelebt wird und das Alter vielmehr in Beziehung zu anderen Altersstufen hergestellt wird.

Für intersektionale Analysen ist insbesondere die Wirkung des erlebten Raums bedeutsam. An welche bestehenden Quartiere das neue Viertel erinnert und welche Bilder damit verknüpft werden, beeinflusst, wer sich von der Konzeption angesprochen fühlt. Aufgrund der plakativen Bildsprache des Wohnprojekts ist es auch denkbar, das Alter im sozialen Aushandlungsprozess kaum eine Rolle spielt, sondern vielmehr die Distinktionen der Lebensstile im Vordergrund stehen. Die Unfertigkeit des Raums erlaubt es, den sozialen Prozess der Aushandlung selbst zu untersuchen und kleinteilig zu entschlüsseln, welche Einflussfaktoren wirksam sind.

\section{Der Raum als methodologische Forschungsstrategie}

Die wachsende Zahl älterer Menschen macht eine Auseinandersetzung mit Alter und Ungleichheit zu einer gesellschaftlich wie wissenschaftlich drängenden Aufgabe. Bislang wird das Alter in der Intersektionalitätsforschung kaum als relevanter Faktor untersucht und in der geographischen Altersforschung spielen Fragen nach Macht und Ungleichheit keine nennenswerte Rolle. Allerdings kann gerade für die $\mathrm{Hu}-$ mangeographie eine Auseinandersetzung mit gesellschaftlicher Ungleichheit und Alterung zu einem fruchtbaren Forschungsfeld werden. Mit diesem Artikel schlagen wir, basierend auf Lefebvre's Raumverständnis, einen methodologischen Zugang zum Zusammenspiel von Ungleichheit und Alter vor, der den Raum als Analyseinstrument des Sozialen begreift. Der Artikel zeigt, dass es für eine fundierte Analyse wichtig ist, den Doppelcharakter von Alter als Kategorie und Prozess anzuerkennen und die Aushandlung beider Facetten in ihrer Räumlichkeit zu denken. Der Raum als Prisma auf Alter(n) bietet einen vielversprechenden Zugang für die Auseinandersetzung mit der Darstellung der Selbstzuschreibung des Attributs ,alt“ nach außen im Altersprozess (1), sowie für die Produktion der Strukturkategorie Alter durch Räu- me (2). Beide Aushandlungsprozesse können nicht für das Alter allein verstanden werden, weil immer auch andere Differenzkategorien eine Wirksamkeit darauf haben, wie Alter wahrgenommen, dargestellt und verhandelt wird.

Über die Altersforschung hinaus sehen wir in dem Ansatz, den Raum als Prisma auf Intersektionalität und Ungleichheit zu verstehen, eine neue Möglichkeit mit der Vielschichtigkeit von Intersektionalität zu arbeiten. Forschung zu Intersektionalität und Ungleichheit muss mit einer enormen Fülle an Komplexität umgehen, was die Konsequenz hat, dass entweder die Konzentration auf wenige bestimmte Differenzmerkmale gelegt wird oder die komplexen Verschränkungen am Fallbeispiel eines Individuums dargelegt werden (McCall, 2005). Anstatt von einem Differenzmerkmal oder einer Situation der Unterdrückung auszugehen, schlagen wir den Raum als methodologischen Ausgangspunkt vor. Keine soziale Situation spielt sich außerhalb des Raums ab - und es gibt keinen Raum, der nicht sozial geprägt wäre. Diese Bedingtheit bzw. Relationalität der beiden Dimensionen erlaubt es, durch das eine Phänomen auf das andere zu schauen. Deshalb plädieren wir für eine methodologische Raumperspektive in der Ungleichheitsforschung. Über die Räumlichkeit des Sozialen lassen sich bestimmbare Orte eingrenzen und die Erkundung der Verflechtungen kann von dort ausgehend beginnen. Für das Alter können so über konkrete Orte die strategische Darstellung der eigenen Alterszuschreibung verstanden, Einblicke über die Aushandlungsprozesse von Alter gewonnen und die Produktion von Alter als Strukturkategorie in den Blick genommen werden. Der Raum als Ausgangspunkt reduziert zudem die Tendenz der Priorisierung eines Differenzmerkmals. Von einem konkreten Ort ausgehend, lässt sich offen fragen, welche Kategorie eine besondere Wirkmacht entfaltet. Ebenso ist die Offenheit für die Entdeckung neuer Ursachen für strukturelle Ungleichheiten gegeben, die eine Forschungsagenda entlang der etablierten Kategorien nur schwer sichtbar machen kann. Je nach zu untersuchendem Raum eröffnet sich der Blick aus einer Mikrooder Makroperspektive. Die eigene Wohnung wird eher Einblicke auf der Mikroebene erlauben, wohingegen institutionalisierte Orte, wie das Altersheim, Ansatzpunkte für die Analyse der gesellschaftlichen Aushandlung von Alter bieten. Der Ausgangspunkt der Analysen kann so vielfältig sein, wie es die Räume sind: das Zuhause, Orte der Freizeitaktivität, Orte des Ehrenamts, der öffentliche Raum, Krankenhäuser, öffentliche Verkehrsmittel oder religiöse Orte. Sie alle bieten einen Anfang, um die Konstruktion und Aushandlung von Alter als Kategorie und Prozess und das Zusammenwirken mit anderen Differenzkategorien zu beforschen.

Datenverfüg barkeit. Für diesen Artikel wurden keine Datensätze genutzt. 
Interessenkonflikt. Die Autorinnen erklären, dass kein Interessenskonflikt besteht.

Danksagung. Die Autorinnen danken zwei anonymen Reviewer*innen sehr herzlich für ihre konstruktive Kritik an früheren Versionen des Artikels. Der vorliegende Beitrag entstand im Rahmen des durch die Deutsche Forschungsgemeinschaft (DFG) geförderten Projekts „Superdiversität und alternde Städte? Die Konvergenz von wachsender Multiethnizität und einer alternden Bevölkerung“ (Projektnummer HE 2417/16-1).

Edited by: Benedikt Korf

Reviewed by: two anonymous referees

\section{Literatur}

Andrews, G. J., Milligan, C., Phillips, D., and Skinner, M.: Geographical gerontology: Mapping a disciplinary Intersection, Geography Compass, 3, 1641-1659, 2009.

Baykara-Krumme, H., Motel-Klingebiel, A., and Schimany, P. (Hrsg.): Viele Welten des Alterns: Ältere Migranten im alternden Deutschland, Alter(n) und Gesellschaft, Springer VS, Wiesbaden, 22, 470 pp., 2012.

Butler, R. N.: Ageism - another form of bigotry, Gerontologist, 9, 243-246, 1969.

Calasanti, T. M.: Incorporating Diversity: Meaning, Levels of Research and Implications for Theory, Gerontologist, 36, 147-156, 1996.

Calasanti, T. and Sleevin, K. (Hrsg.): Age Matters: Realigning Feminist Thinking, Routledge, New York, USA, 2006.

Calasanti, T., Sleevin, K., and King, N.: Ageism and Feminism: From "et cetera" to center, NWSA Journal, 18, 13-30, 2006.

Clemens, W.: Zur „ungleichheitsempirischen Selbstvergessenheit, der deutschsprachigen Alter(n)ssoziologie, in: Soziale Ungleichheiten und kulturelle Unterschiede in Lebenslauf und Alter: Fakten, Prognosen und Visionen, Herausgeber: Künemund, H. and Schroeter, K. R., Alter(n) und Gesellschaft, 15, VS Verlag für Sozialwissenschaften | GWV Fachverlage GmbH, Wiesbaden, 17-30, 2008.

Crenshaw, K.: Demarginalizing the intersection of race and sex: A black feminist critique of antidiscrimination doctrine, feminist theory and antiracist politics, Univ. Chicago Leg. For., USA, 139-167, 1989.

Cronin, A. and King, A.: Power, Inequality and Identification: Exploring Diversity and Intersectionality amongst Older LGB Adults, Sociology, 44, 876-892, 2010.

Dannefer, D.: Cumulative advantage/disadvantage and the life course: Cross-fertilizing age and social science theory, J. Gerontol. B-Psychol., 58, S327-S337, 2003.

Denninger, T. and Schütze, L. (Hrsg.): Alter(n) und Geschlecht: Neuverhandlungen eines sozialen Zusammenhangs, Forum Frauen- und Geschlechterforschung, Westfälisches Dampfboot, Münster, 47, 2017.

Estes, C.: The Ageing Enterprise, Jossey-Bass, San Francisco, USA, 1979.
Franz, P. and Vaskovics, L.: Die räumliche Segregation alter Menschen in bundesdeutschen Städten, Z. Gerontologie, 15, 280287, 1982.

GESOBAU: Innovative Wohnkonzepte erleben, online abrufbar: https://www.gesobau.de/mieterservice/wohnkonzepte/ pflegequartier.html, letzter Zugriff: 7 May 2018.

GSW: Future Living, Presseinformation, online abrufbar: https://future-living-berlin.com/img/2017/08/GSW_ Presseinformation_Future_Living.pdf, letzter Zugriff: 7 May 2018.

Hagestad, G. and Uhlenberg, P.: The social separation of old and young: A root of ageism, J. Soc. Issues, 61, 343-360, 2005.

Harper, S.: Constructing later life/constructing the body: some thoughts from feminist theory, in: Critical approaches to ageing and later life, Herausgeber: Jamieson, A., Harper, S., and Victor, C., Open University Press, Buckingham, 160-172, 1997.

Harper, S. and Laws, G.: Rethinking the Geography of Ageing, Prog. Hum. Geog., 19, 199-221, 1995.

Havemann, M. and Stöppler, R.: Altern mit geistiger Behinderung: Grundlagen für die Begleitung, Bildung und Rehabilitation, Kohlhammer, Stuttgart, 2009.

Helbrecht, I.: Wohneigentum statt Rente? Demographischer Wandel und Altersvorsorge in acht europäischen Ländern im Vergleich, in: Informationen zur Raumentwicklung, 197-210, 2013.

Helbrecht, I. and Geilenkeuser, T.: Demographischer Wandel, Generationeneffekte und Wohnungsmarktentwicklung - Wohneigentum als Altersvorsorge?, in: Raumforschung und Raumordnung 70, 425-436, https://doi.org/10.1007/s13147-012-0185-8, 2012.

Henery, C. S.: Where they Walk: What Aging Black Women's Geographies Tell of Race, Gender, Space, and Social Transformation in Brazil, Cultural Dynamics, 23, 85-106, 2011.

Hopkins, P. and Pain, R.: Geographies of age: Thinking relationally, AREA, 39, 287-294, https://doi.org/10.1111/j.14754762.2007.00750.x, 2007.

Höppner, G.: Non-verbale (Neu-)Verhandlungen der Geschlechtsspezifischen Körperoptimierungsdebatte im Alter am Beispiel des Imperativa „Sei schlank!“, in: Alter(n) und Geschlecht: Neuverhandlungen eines sozialen Zusammenhangs, Herausgeber: Denninger, T. and Schütze, L., Forum Frauen- und Geschlechterforschung, Westfälisches Dampfboot, Münster, 47, 190-210, 2017.

Khan-Zvornicanin, M.: Kultursensible Altenhilfe?: Neue Perspektiven auf Programmatik und Praxis gesundheitlicher Versorgung im Alter, transcript Verlag, Bielefeld, 2016.

Klaus, D. and Baykara-Krumme, H.: Die Lebenssituation von Personen in der zweiten Lebenshälfte mit und ohne Migrationshintergrund, in: Altern im Wandel: Zwei Jahrzehnte Deutscher Alterssurvey (DEAS), Herausgeber: Mahne, K., Wolff, J. K., Simonson, J., and Tesch-Römer, C., Springer Fachmedien, Wiesbaden, 359-379, 2017.

Kohli, M.: Ageing as a Challenge for Sociological Theory, Ageing Soc., 8, 367-394, https://doi.org/10.1017/S0144686X00007169, 1988.

Kohli, M.: Das Alter als Herausforderung für die Theorie sozialer Ungleichheit, in: Lebenslagen, Lebensläufe, Lebensstile, Herausgeber: Berger, P. and Hradil, S., Schwartz \& Co, Göttingen, 387-406, 1990.

Kohli, M., Künemund, H., Motel, A., and Sydlik, M.: Soziale Ungleichheit, in: Die zweite Lebenshälfte. Gesellschaftliche Lage 
und Partizipation im Spiegel des Alters-Survey, Herausgeber: Kohli, M. and Künemund, H., Leske \& Budrich, Opladen, 337342, 2000.

Krekula, C.: The Intersection of Age and Gender, Curr. Sociol., 55, 155-171, https://doi.org/10.1177/0011392107073299, 2007.

Künemund, H. and Schroeter, K. R. (Hrsg.): Soziale Ungleichheiten und kulturelle Unterschiede in Lebenslauf und Alter: Fakten, Prognosen und Visionen, Alter(n) und Gesellschaft, VS Verlag für Sozialwissenschaften | GWV Fachverlage GmbH, Wiesbaden, 15, 2008.

Lefebvre, H.: The production of space, vol. 142, Blackwell, Oxford, 1991.

Lejeune, C., Romeu Gordo, L. and Simonson, J.: Einkommen und Armut in Deutschland: Objektive Einkommenssituation und deren subjektive Bewertung, in: Altern im Wandel: Zwei Jahrzehnte Deutscher Alterssurvey (DEAS), Herausgeber: Mahne, K., Wolff, J. K., Simonson, J., and Tesch-Römer, C., Springer Fachmedien, Wiesbaden, 97-110, 2017.

Linschoten, M., Lottmann, R., and Lauscher, F.: „'The Pink Passkey' ${ }^{\circledR}$ - ein Zertifikat für die Verbesserung der Akzeptanz von LSBT* I-Pflegebedürftigen in Pflegeeinrichtungen. “ In Homosexualität_en und Alter (n), Springer VS, Wiesbaden, 227-241, 2016.

Lottmann, R., Lautmann, R., and Castro Varela, M. d. M. (Hrsg.): Homosexualität_en und Alter(n), Springer Fachmedien, Wiesbaden, 2016.

Lutz, H. and Wenning, N.: Differenzen über Differenz - Einführung in die Debatten, in: Unterschiedlich verschieden: Differenz in der Erziehungswissenschaft, Herausgeber: Lutz, H. and Wenning, N., VS Verlag für Sozialwissenschaften, Wiesbaden, 1124, 2001.

Mahne, K., Wolff, J. K., Simonson, J., and Tesch-Römer, C. (Hrsg.): Altern im Wandel: Zwei Jahrzehnte Deutscher Alterssurvey (DEAS), Springer Fachmedien, Wiesbaden, 2017.

Matthäi, I.: Die ,vergessenen“ Frauen aus der Zuwanderergeneration: zur Lebenssituation von alleinstehenden Migrantinnen im Alter, VS Verlag für Sozialwissenschaften, Wiesbaden, 2005.

McCall, L.: The Complexity of Intersectionality, Signs: Journal of Women in Culture and Society, 30, 1771-1800, https://doi.org/10.1086/426800, 2005.

McHugh, K. E.: Generational consciousness and retirement communities, Popul. Space Place, 13, 293-306, https://doi.org/10.1002/psp.448, 2007.

McMullin, J. A.: Theorizing Age and Gender Relations, in: Connecting Gender and Ageing, Herausgeber: Arber, S. and Ginn, J., Buckingham/Philadelphia, 30-41, 1995.

McMullin, J. A.: Diversity and the State of Sociological Aging Theory, Gerontologist, 40, 517-530, https://doi.org/10.1093/geront/40.5.517, 2000.

Merrell, J., Kinsella, F., Murphy, F., Philpin, S., and Ali, A.: Accessibility and equity of health and social care services: exploring the views and experiences of Bangladeshi carers in South Wales, UK, Health Soc. Care Comm., 14, 197-205, https://doi.org/10.1111/j.1365-2524.2006.00610.x, 2006.

Milligan, C.: There's no place like Home: Place and care in an Ageing Society, Geographies of Health, Ashgate, Farnham, 2009.
Mowl, G., Pain, R., and Talbot, C.: The ageing body and the homespace, AREA, 32, 189-197, https://doi.org/10.1111/j.14754762.2000.tb00129.x, 2000.

Nowossadeck, S. and Mahne, K.: Bewertung des Wohnumfeldes in der zweiten Lebenshälfte, in: Altern im Wandel: Zwei Jahrzehnte Deutscher Alterssurvey (DEAS), Herausgeber: Mahne, K., Wolff, J. K., Simonson, J., and Tesch-Römer, C., Springer Fachmedien, Wiesbaden, 301-314, 2017.

Oswald, F., Kaspar, R., Frenzel-Erkert, U. and Konopik, N.: „Hier will ich Wohnen bleiben!“": Ergebnisse eines Frankfurter Forschungsprojekts zur Bedeutung des Wohnens in der Nachbarschaft für gesundes Altern, Frankfurt am Main, 2013.

Pain, R., Mowl, G., and Talbot, C.: Difference and the Negotiation of "Old Age", Environ. Plann. D., 18, 377-393, https://doi.org/10.1068/d31j, 2000.

Pain, R., Barke, M., Gough, J., Fuller, D., MacFarlane, R., and Mowl, G.: Introducing social geographies, Arnold, London, 2001.

Rohstock, N.: Altersbilder und Lebenssituationen: Vergleichende Untersuchungen zu Türkinnen und Türken in Deutschland und in der Türkei, Zugl.: Freiburg, Univ., Diss., 2013, Internationale Hochschulschriften, 606, Waxmann, Münster, 2014.

Ronneberger, K. and Vogelpohl, A.: Henri Lefebvre: Die Produktion des Raumes und die Urbanisierung der Gesellschaft, Herausgeber: Oßenbrügge, J. und Vogelpohl, A.: Theorien in der Raum-und Stadtforschung. Einführungen, Westfälisches Dampfboot, Münster, 251-270, 2014.

Rothfuß, E. and Gerhard, U.: Urbane Ungleichheit in vergleichender Perspektive - Konzeptionelle Überlegungen und empirische Befunde aus den Americas, Geogr. Helv., 69, 67-78, https://doi.org/10.5194/gh-69-67-2014, 2014.

Scherger, S., Nazroo, J., and Higgs, P.: Leisure activities and retirement: Do structures of inequality change in old age?, Ageing Soc., 31, 146-172, https://doi.org/10.1017/S0144686X10000577, 2011.

Schneider-Sliwa, R.: Städtische Umwelt im Alter: Präferenzen älterer Menschen zum altersgerechten Wohnen, zur Wohnumfeldund Quartiersgestaltung in Basel, Geogr. Helv., 59, 300-312, https://doi.org/10.5194/gh-59-300-2004, 2004.

Schütze, L.: Vergessene „Verwobenheiten“: Alter(n) und Homosexualität als intersektionale Subjektordnungen, in: Alter(n) und Geschlecht: Neuverhandlungen eines sozialen Zusammenhangs, Herausgeber: Denninger, T. and Schütze, L., Forum Frauen- und Geschlechterforschung, Westfälisches Dampfboot, Münster, 47, 131-149, 2017.

Schwanen, T., Hardill, I., and Lucas, S.: Spatialities of ageing: The co-construction and co-evolution of old age and space, Geoforum, 43, 1291-1295, https://doi.org/10.1016/j.geoforum.2012.07.002, 2012.

Skinner, M. W., Cloutier, D., and Andrews, G. J.: Geographies of ageing: Progress and possibilities after two decades of change, Prog. Hum. Geog., 39, 776-799, https://doi.org/10.1177/0309132514558444, 2015.

Slevin, K.: The Embodied Experiences of Old Lesbians, in: Age Matters: Realigning Feminist Thinking, Herausgeber: Calasanti, T. and Sleevin, K., Routledge, New York, 247-268, 2006.

Sontag, S.: The double standard of ageing, in: On the Contrary: Essays by Men and Women, Herausgeber: Rainbolt, M. and 
Fleetwood, J., Saturday Review of the Society, 55, State University of New York Press, Albany, 29-38, 1972.

Stoller, E. P. and Gibson, R. C.: Worlds of Difference: Inequality in the Aging Experience, 3rd edn., SAGE Publications, Thousand Oaks, 377 pp., 1994.

Townsend, P.: The Structured Dependency of the Elderly: A Creation of Social Policy in the Twentieth Century, Ageing Soc., 1, 5-28, https://doi.org/10.1017/S0144686X81000020, 1981.

Traunsteiner, B. S.: Gleichgeschlechtlich liebende Frauen im Alter: Intersektionalität, Lebenslagen und Antidiskriminierungsempfehlungen, Springer Fachmedien, Wiesbaden, 2018.

Tucci, I. and Yildız, S.: Das Alterseinkommen von Migrantinnen und Migranten: zur Erklärungskraft von Bildungs-und Erwerbsbiografien, in: Viele Welten des Alterns: Ältere Migranten im alternden Deutschland, Herausgeber: Baykara-Krumme, H., Motel-Klingebiel, A., and Schimany, P., Alter(n) und Gesellschaft, Springer VS, Wiesbaden, 22, 101-126, 2012.

Twigg, J.: The body, gender, and age: Feminist insights in social gerontology, J. Aging Stud., 18, 59-73, https://doi.org/10.1016/j.jaging.2003.09.001, 2004.

Valentine, G.: Public space and the culture of childhood, Ashgate, Aldershot, 2004.

Valentine, G.: Theorizing and Researching Intersectionality: A Challenge for Feminist Geography, Prof. Geogr., 59, 10-21, https://doi.org/10.1111/j.1467-9272.2007.00587.x, 2007.

van Dyk, S.: Soziologie des Alters, Einsichten Themen der Soziologie, transcript-Verlag, Bielefeld, 2015a. van Dyk, S.: Vom Leben in der Zeit.: Theoretische Perspektiven auf die Analyse von Lebensalter und die gesellschaftliche Norm der Alterslosigkeit, Zeitschrift für theoretische Soziologie, 4, 208235, 2015 b.

van Dyk, S.: Zur Interdependenz und Analyse von Alter(n) und Geschlecht.: Theoretische Erkundungen und zeitdiagnostische Überlegungen, in: Alter(n) und Geschlecht: Neuverhandlungen eines sozialen Zusammenhangs, Herausgeber: Denninger, T. and Schütze, L., Forum Frauen- und Geschlechterforschung, Westfälisches Dampfboot, Münster, 47, 24-50, 2017.

Vincent, J. A.: Inequality and old age, UCL Press, London, 218 pp., 1995.

Vogel, C. and Motel-Klingebiel, A. (Hrsg.): Altern im sozialen Wandel: Die Rückkehr der Altersarmut?, Alter(n) und Gesellschaft, Springer VS, Wiesbaden, 23, 2013.

Willson, A. E., Shuey, K. M., and Elder Jr., G. H.: Cumulative advantage processes as mechanisms of inequality in life course health, Am. J. Sociol., 112, 1886-1924, 2007.

Winkler, G. and Degele, N.: Intersektionalität: Zur Analyse sozialer Ungleichheiten, 2. unveränderte Auflage, transcript Verlag, Bielefeld, 2010.

Wray, S.: Women growing older: Agency, Ethnicity and Culture, Sociology, 37, 511-527, 2003. 